\title{
DIAGNÓSTICO DE LA GESTIÓN PÚBLICA DEL TERRITORIO COSTERO DEL PAÍS VASCO
}

\author{
Ibone Santos-Ellakuria \\ Área de Análisis Geográfico Regional, Universidad de Cádiz \\ Departamento de Geografía, Prehistoria y Arqueología, \\ Universidad del País Vasco (UPV/EHU) \\ C/ Tomás y Valiente s/n, 01006 Vitoria-Gasteiz \\ ibone.santos@ehu.eus
}

\begin{abstract}
Resumen: Las áreas litorales tienen una especial relevancia en la ordenación territorial debido a su importancia socioeconómica y ambiental y a su elevada exposición a impactos derivados de actividades humanas y riesgos naturales. La gestión integrada de zonas costeras (GIZC) tiene como objetivo el desarrollo sostenible de las zonas costeras, integrando las políticas sectoriales y los componentes terrestres y marinos del litoral. Se realiza un diagnóstico del marco jurídico-administrativo de la gestión del litoral del País Vasco, con el fin de detectar aquellas disfunciones que sería preciso subsanar para desarrollar una política de GIZC. Dicho diagnóstico se realiza mediante el análisis de las políticas, normativas vigentes, competencias administrativas, instrumentos de planificación, programas de formación, grado de conocimiento del medio y mecanismos de participación en la toma de decisiones. Se concluye que para desarrollar una política regional de GIZC es necesario aumentar la integración y coordinación de las políticas sectoriales y mejorar la gobernanza.
\end{abstract}

Palabras clave: Gestión integrada de zonas costeras (GIZC), marco jurídico-administrativo, planificación integral, desarrollo sostenible, Comunidad Autónoma del País Vasco.

Abstract: Coastal zones have special relevance in spatial planning due to their socioeconomic and environmental importance and their exposure to the impacts of human activities and natural risks. The objective of integrated coastal zone management (ICZM) is the sustainable development

Recibido: 28-01-2019. Aceptado: 02-04-2019. 
of coastal zones, integrating sectoral policies and the coastal and marine components of the coast. A diagnosis of the legal-administrative framework of the management of the Basque Country is made, in order to detect those dysfunctions that are needed to correct to develop an ICZM policy. The diagnosis is made through the analysis of policies, current regulations, administrative competences, planning instruments, training programs, degree of knowledge of the environment and mechanisms of participation in decision making. It is concluded that to develop a regional ICZM policy, it is necessary to increase the integration and coordination of sectoral policies and improve governance.

Keywords: Integrated coastal zone management (ICZM), legal and administrative framework, comprehensive planning, sustainable development, Autonomous Community of the Basque Country.

\section{Introducción}

Las zonas marinas y costeras están sometidas a importantes presiones derivadas de las actividades humanas. Estas presiones pueden desembocar en la degradación de los servicios de los ecosistemas litorales y crear un impacto significativo en el desarrollo y el crecimiento económico (Cicin-Sain, 1993). Para prevenir y solventar dichos impactos es imprescindible buscar estrategias que den respuesta a las causas originales de los problemas socio-ambientales de las áreas litorales (Clark, 1995; Post y Lundin, 1996).

Una forma adecuada de asegurar su conservación y uso sostenible la constituye la gestión integrada de zonas costeras (GIZC) (Post y Lundin, 1996), también denominada gestión integrada de áreas litorales (Barragán, 2004; Pérez-Cayeiro, 2013). La GIZC puede considerarse como un modelo de gestión novedoso que ha eclosionado a partir de los años noventa del siglo XX y que ofrece consenso, tanto a nivel europeo como internacional, para revertir la actual tendencia negativa del espacio costero. Se trata de un proceso dinámico, continuo e interactivo destinado a promover el desarrollo sostenible de las zonas costeras mediante la integración de políticas, objetivos, estrategias y planes sectoriales en el espacio y el tiempo y la integración de los componentes terrestres y marinos del litoral (Cicin-Sain, 1993; Post y Lundin, 1996; Barragán, 2004; Pérez-Cayeiro, 2013; García-Sanabria, 2015; Barragán y de Andrés, 2016; Ahlhorn, 2018).

La Recomendación 2002/413/CE del Parlamento Europeo y del Consejo de la Unión Europea (UE) (2002), relativa a la aplicación de la gestión integrada de las zonas costeras en Europa, instó a los Estados miembros a desarrollar un marco legal adecuado y mecanismos para una correcta gestión de las zonas costeras a través de la elaboración de una estrategia estatal. En el Estado español, en respuesta a las recomendacio- 
nes del Parlamento Europeo y del Consejo, se redactó una estrategia estatal para la GIZC (Gobierno de España, 2006), la cual sin embargo no ha sido tomada en cuenta a nivel político (Barragán, 2010). A escala autonómica, han sido cuatro las Comunidades Autónomas que han redactado su estrategia regional de GIZC (Asturias, Cataluña, Valencia y Andalucía), aunque en ninguna de ellas se ha comenzado a ejecutar (Farinós, 2011).

Esta carencia de avances en materia de GIZC en las costas españolas parece estar en consonancia con las escasas referencias sobre gestión integrada de las costas que recoge la vigente Directiva 2014/89/UE, por la que se establece un marco para la ordenación del espacio marítimo de la Unión Europea (Parlamento Europeo y Consejo de la UE, 2014). Esta Directiva 2014/89/UE (a diferencia de la mencionada Recomendación 2002/413/CE y de la Propuesta de Directiva para establecer un marco para la ordenación del espacio marítimo y la gestión integrada de las costas emitida por la Comisión Europea en 2013), se centra en la ordenación del espacio marítimo y no establece una diferenciación respecto a la gestión de las zonas costeras. Así, la Directiva 2014/89/UE se limita a instar a los Estados miembros a procurar promover la coherencia entre la ordenación del espacio marítimo y otros procesos como la gestión integrada de las costas o prácticas formales o informales equivalentes.

\section{1. Área de estudio}

La franja litoral de la Comunidad Autónoma del País Vasco (CAPV) se ubica en las provincias de Bizkaia y Gipuzkoa y presenta una longitud de $250 \mathrm{~km}$, de los cuales 150 $\mathrm{km}(60 \%)$ corresponden a acantilados, $70 \mathrm{~km}(28 \%)$ a estuarios, marismas y áreas urbanizadas y $30 \mathrm{~km}(12 \%)$ a sectores de arena (Iglesias et al., 2018). El área de estudio comprende 62 municipios, repartidos en 6 áreas funcionales de carácter supramunicipal sobre las que se realiza la planificación territorial subregional, y que reciben el nombre del municipio designado como cabecera (Gobierno Vasco, 1997) (Figura 1). El territorio costero coincide con el que contempla el Plan Territorial Sectorial de Protección y Ordenación del Litoral (PTSL) (Gobierno Vasco, 2007), incluyendo el medio marino litoral, que comprende el espacio entre la línea de bajamar escorada o máxima viva equinoccial y la isobata de $50 \mathrm{~m}$ (Figura 1).

La elevada concentración poblacional, de infraestructuras y de actividades económicas en ciertos sectores de la abrupta costa vasca genera relevantes impactos a tener en cuenta por la GIZC. Uno de los principales problemas derivados de las actividades humanas es la degradación de los ecosistemas litorales (Gobierno Vasco, 2015, 2016c). Asimismo, la costa vasca es proclive a parecer episodios de inundaciones y deslizamientos, así como eventos de fuerte oleaje (Gaztelumendi et al., 2018). Estos riesgos naturales se ven agravados por el aumento de la urbanización, la regulación de cauces fluviales y los dragados. A todo ello hay que sumar los efectos del cambio climático en el litoral, como el ascenso del nivel del mar y el incremento de los episodios de gran oleaje (Chust et al., 
2011, Marcos et al., 2012). Además, la ocupación de las dunas por urbanizaciones o por paseos marítimos, la construcción de espigones y los dragados y deposiciones han modificado la dinámica litoral (Puig y Villarroya, 2013; Monge-Ganuzas et al., 2019). Otro de los problemas a tener en cuenta por la GIZC es la contaminación de aguas, si bien los informes de seguimiento de las masas de agua costeras de la CAPV muestran una evolución de mejoría en este aspecto (Borja et al., 2018). También es preciso una ordenación integrada para aspectos como la selección de ubicaciones idóneas para instalaciones de captación de energía del oleaje (Galparsoro et al., 2012) o la planificación sostenible del turismo (Torres-Delgado y Saarinen, 2014; Iglesias et al., 2018).

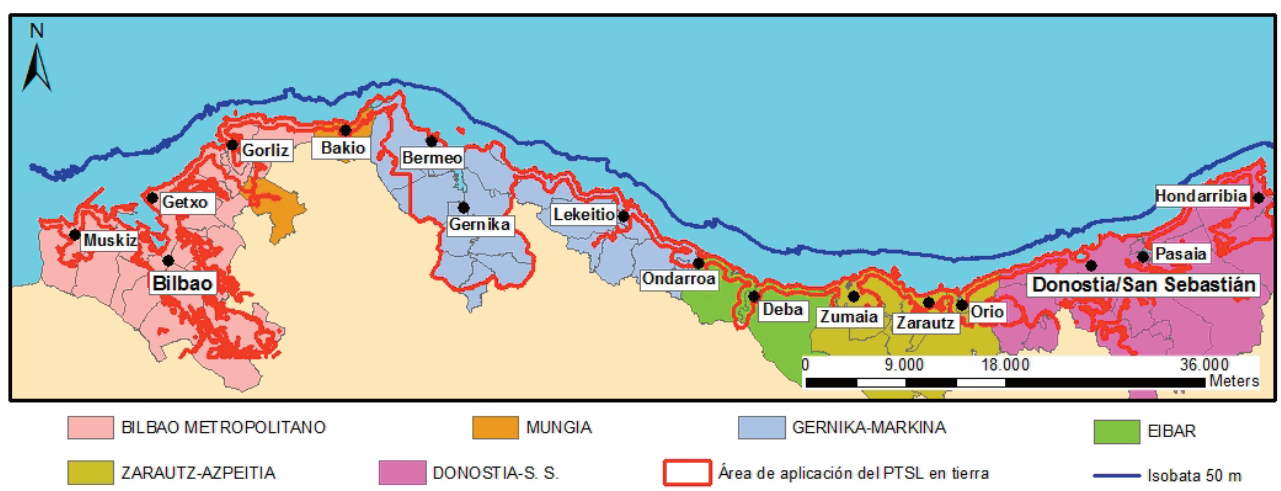

Figura 1. Mapa de los límites del área de estudio y de los municipios y áreas funcionales incluidos en la misma. Se incluye la ubicación de los principales núcleos de población.

Fuente: Elaboración propia a partir de la Infraestructura de Datos Espaciales de Euskadi.

\subsection{Objetivos}

El objetivo que se persigue es realizar un diagnóstico del actual sistema jurídico-administrativo de la gestión costera de la CAPV que ayude a comprender sus disfunciones. De esta forma, con el presente diagnóstico se pretende contribuir a sentar unas bases para el futuro diseño de una política de GIZC en el territorio litoral del País Vasco.

A modo de objetivos específicos se pretende: i) abordar la política pública y la normativa vasca en materia de gestión costera; ii) analizar los instrumentos estratégicos y operativos que mayor repercusión tienen en la gestión del litoral vasco; iii) estudiar la estructura institucional y competencial de la gestión de recursos costeros, iv) determinar el grado de formación, conocimiento, educación y participación de los agentes vinculados al litoral. 


\section{Metodología}

El modelo metodológico utilizado se basa en la realización de un análisis de los aspectos más relevantes para la gestión de áreas litorales en la CAPV desde el punto de vista jurídico-administrativo (política y estrategia, normativa, competencias, instituciones, instrumentos, formación y capacitación, recursos económicos, información y conocimiento, educación para la sostenibilidad y participación) (Barragán, 2004). Es preciso señalar que no se han considerado los recursos económicos destinados a la gestión del litoral del País Vasco, debido a la dificultad de acceso a los datos específicos referidos a dicha gestión.

En segundo lugar, considerando conjuntamente los distintos aspectos analizados del subsistema jurídico-administrativo, se realiza un análisis DAFO (Barragán, 2004; Sanò et al., 2010) en el que se sintetizan las debilidades y fortalezas del marco interno de la gestión pública de la costa vasca, así como las amenazas y oportunidades del contexto externo.

\section{Resultados}

\subsection{Política costera}

La ejecución de políticas públicas en relación con la gestión del litoral del País Vasco ha ido orientada a resolver los problemas generales derivados del contexto socioeconómico. En este sentido, tradicionalmente los conflictos de la franja costera han sido tratados desde distintas perspectivas sectoriales (medio ambiente, turismo, pesca, transporte, etc.) y no ha existido una política explícita de acción integrada. Solo algunas de las políticas han concretado acciones o planes integrados, como la de medio ambiente y la de ordenación del territorio. A continuación, se hace una breve descripción de la evolución de ambas.

Las prioridades de actuación de la política ambiental vasca (PAV) se han establecido en función de las principales presiones ambientales derivadas del sistema productivo. De esta forma, la contaminación atmosférica, el cambio climático, los suelos contaminados, la gestión de los residuos o la calidad de las aguas, unidas a los conflictos socio-ambientales causados por estos problemas han contribuido a marcar las prioridades de la PAV (Arto, 2010).

La preocupación política por los temas ambientales comenzó a ser notoria a mediados de los años noventa del siglo XX (García-Sanabria et al., 2011). Dicha preocupación se ve plasmada en la aprobación de varios instrumentos normativos y en el uso del término "medio ambiente" para denominar un Departamento del Gobierno Vasco. A partir de la quinta legislatura (1995-1999) aparece por primera vez un Departamento que liga el medio ambiente y la ordenación del territorio. Desde entonces ambas políticas se han situado en un mismo Departamento hasta la actualidad. 
La CAPV cuenta desde 1990 con la Ley 4/1990 de Ordenación del Territorio del País Vasco, que preveía la elaboración de un Plan Territorial Sectorial del Litoral de la CAPV. No obstante, hubo que esperar diecisiete años para que se aprobara el PTSL (Gobierno Vasco, 2007). Este instrumento constituye el marco que integra la política territorial de la costa vasca y entiende que el litoral está formado por el espacio terrestre, los medios de transición y el medio marino.

Respecto a la adopción de políticas europeas y al contrario que otras CCAA (Asturias, Cataluña, Valencia y Andalucía), la CAPV no ha redactado ninguna estrategia de GIZC, tal y como se instaba en la Recomendación 2002/413/CE del Parlamento Europeo. Si bien con el PTSL se han dado importantes avances, sigue sin existir una política vasca específica para la GIZC. Prácticamente todas las políticas sectoriales tienen impacto en la zona costera: desarrollo económico, transporte, vivienda, energía, turismo, agricultura y pesca, etc. Así, la gestión del litoral vasco ha sido regida por criterios sectoriales, siendo la ordenación del territorio plasmada en el PTSL el planteamiento más integrador existente hasta el momento.

\subsection{Normativa}

El panorama legislativo que afecta al ámbito costero-marino vasco está compuesto por un complejo conjunto de instrumentos legales (Tabla 1). A nivel estatal, el instrumento normativo principal del que se sirve la Administración para la ordenación, protección y defensa del litoral es la Ley 22/1988 de Costas modificada por la Ley 2/2013 de protección y uso sostenible del litoral. En la Ley de Costas se definen el Dominio Público Marítimo Terrestre (DPMT), así como sus limitaciones de uso y las de los terrenos colindantes, y las servidumbres legales: la Servidumbre de Protección (100 m ampliables a 200 m por la propia Administración del Estado de acuerdo con la Comunidad Autónoma y los Ayuntamientos correspondientes), la Servidumbre de Tránsito (6 m ampliables hasta $20 \mathrm{~m}$ ) y la Zona de Influencia $(500 \mathrm{~m})$. Esta Ley establece un marco jurídico común para la gestión del DPMT de todo el Estado y sus determinaciones son acatadas por la estructura normativa propia de la CAPV.

En la escala autonómica es relevante la Ley 1/2006 de Aguas (Tabla 1) pues contempla los aportes que llegan al mar. Por un lado, determina los objetivos medioambientales y regula la protección y utilización de las aguas continentales, costeras y de transición. Por otro lado, establece los regímenes de planificación en materia de aguas y obras hidráulicas. Además, define normas generales de abastecimiento, saneamiento, depuración y riego. A este respecto destaca la obligación de los ayuntamientos de reglar mediante ordenanza local el régimen jurídico del servicio de saneamiento y depuración de aguas. 
Tabla 1. Normativas que afectan a la gestión de las zonas costeras de la CAPV

\begin{tabular}{|c|c|c|}
\hline & Recurso/actividad regulada & Referencia normativa \\
\hline \multirow{3}{*}{ 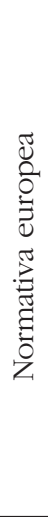 } & Aguas & $\begin{array}{l}\text { Directiva 2000/60/CE del Parlamento Europeo y del } \\
\text { Consejo por la que se establece un marco comunitario } \\
\text { de actuación en el ámbito de la política de aguas } \\
\text { (Directiva marco sobre el agua). }\end{array}$ \\
\hline & $\begin{array}{l}\text { Conservación de } \\
\text { la naturaleza }\end{array}$ & $\begin{array}{l}\text { Directiva 92/43/CEE del Consejo relativa a la conservación } \\
\text { de los hábitats naturales y de la fauna y flora silvestres } \\
\text { (Directiva de hábitats). } \\
\text { DIRECTIVA 2009/147/CE del Parlamento Europeo y del } \\
\text { Consejo relativa a la conservación de las aves silvestres } \\
\text { (Directiva de aves). }\end{array}$ \\
\hline & Litoral & $\begin{array}{l}\text { Directiva 2008/56/CE del Parlamento Europeo y del } \\
\text { Consejo, por la que se establece un marco de acción } \\
\text { comunitaria para la política del medio marino } \\
\text { (Directiva marco sobre la estrategia marina). }\end{array}$ \\
\hline \multirow{5}{*}{ 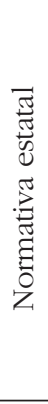 } & Aguas & $\begin{array}{l}\text { Real Decreto Legislativo } 1 / 2001 \text { por el que se aprueba } \\
\text { el texto refundido de la Ley de Aguas. }\end{array}$ \\
\hline & $\begin{array}{l}\text { Conservación de } \\
\text { la naturaleza }\end{array}$ & $\begin{array}{l}\text { Ley 42/2007 del Patrimonio Natural y de la Biodiversidad. } \\
\text { Real Decreto 556/2011 para el desarrollo del Inventario } \\
\text { Español del Patrimonio Natural y la Biodiversidad. }\end{array}$ \\
\hline & Litoral & Ley $2 / 2013$ de protección y uso sostenible del litoral. \\
\hline & Pesca & Ley 3/2001 de Pesca Marítima del Estado. \\
\hline & $\begin{array}{l}\text { Puertos y asuntos } \\
\text { marítimos }\end{array}$ & $\begin{array}{l}\text { Real decreto Legislativo } 2 / 2011 \text { por el que se aprueba } \\
\text { el Texto Refundido de la Ley de Puertos del Estado y } \\
\text { de la Marina Mercante. }\end{array}$ \\
\hline \multirow{8}{*}{ 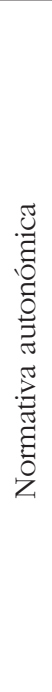 } & Aguas & Ley $1 / 2006$ de Aguas. \\
\hline & $\begin{array}{l}\text { Conservación de } \\
\text { la naturaleza }\end{array}$ & $\begin{array}{l}\text { Ley 16/1994 de conservación de la naturaleza del } \\
\text { País Vasco. } \\
\text { Ley } 5 / 1989 \text { de protección y ordenación de la Reserva } \\
\text { de la Biosfera de Urdaibai. }\end{array}$ \\
\hline & Impacto ambiental & $\begin{array}{l}\text { Ley 3/1998 general de protección del medio ambiente } \\
\text { del País Vasco. } \\
\text { Decreto } 183 / 2003 \text { por el que se regula el procedimiento } \\
\text { de evaluación conjunta de impacto ambiental. }\end{array}$ \\
\hline & \begin{tabular}{|l} 
Ordenación del Territorio y \\
del litoral, suelo y urbanismo
\end{tabular} & $\begin{array}{l}\text { Ley 4/1990 de Ordenación del Territorio del País Vasco. } \\
\text { Ley 2/2006 del Suelo y Urbanismo del País Vasco. }\end{array}$ \\
\hline & Paisaje & $\begin{array}{l}\text { Decreto 90/2014 sobre protección, gestión y ordenación } \\
\text { del paisaje en la ordenación del territorio del País Vasco. }\end{array}$ \\
\hline & $\begin{array}{l}\text { Puertos y asuntos } \\
\text { marítimos }\end{array}$ & $\begin{array}{l}\text { Ley 2/2018 de Puertos y Transporte Marítimo del } \\
\text { País Vasco. }\end{array}$ \\
\hline & Pesca & Ley 6/1998 de Pesca Marítima. \\
\hline & Turismo & Ley 6/1994 de Ordenación del Turismo. \\
\hline
\end{tabular}

Fuente: Elaboración propia. 
Por su parte, la Ley 16/1994 de Conservación de la Naturaleza, modificada por la 2/2013, establece el régimen de protección y conservación de los recursos naturales de la CAPV. Para ello se prevén cuatro categorías de espacios naturales protegidos cuya declaración por decreto corresponde al Gobierno Vasco. Además existen otros ámbitos protegidos que disponen de otras regulaciones como los Humedales de Importancia Internacional Ramsar, la Reserva de la Biosfera de Urdaibai o el Geoparque mundial Unesco de la Costa Vasca. En caso de solaparse en un mismo lugar distintas figuras de espacios protegidos, las normas reguladoras de los mismos, así como los mecanismos de planificación se integrarán en un único documento de planificación del espacio.

En relación con el litoral, la Ley 3/1998 de Protección del Medio Ambiente del País Vasco complementa lo establecido en la Ley de Costas: los usos y actividades en la zona de servidumbre de protección del dominio público marítimo-terrestre, así como los vertidos industriales y contaminantes en las aguas territoriales del Estado correspondientes al litoral vasco, estarán sometidos a autorización administrativa del órgano ambiental de la CAPV de acuerdo con la legislación sectorial en la materia y lo que se disponga reglamentariamente. Por otro lado, se define la zona ambientalmente sensible como aquellos espacios que por sus especiales características en cuanto a valores ambientales contenidos y fragilidad de los mismos sea susceptible de un mayor deterioro ambiental. Las zonas ambientalmente sensibles que pueden estar relacionadas con el litoral son el DPMT y la Servidumbre de Protección, el Dominio Público Hidraúlico, las áreas de recarga de acuíferos, las áreas o enclaves de elevado interés naturalístico, y las áreas o enclaves catalogados o inventariados por constituir parte del patrimonio histórico artístico. Se exceptúa de ser considerado zona ambientalmente sensible el territorio clasificado como suelo urbano en el momento de promulgación de dicha Ley, lo cual, como señalan Lazcano y Arzoz (2009), no es muy ortodoxo con arreglo a lo previsto en la Ley de Costas ya que en suelo urbano no desaparece la servidumbre de protección, sino que se limita su extensión territorial de 100 a 20 metros.

Otra norma autonómica de relevancia para la gestión del litoral es la Ley 4/1990 de Ordenación del Territorio del País Vasco, que se configura como el marco de referencia para el resto de políticas sectoriales. Constituye el marco jurídico de la política de ordenación del territorio en la CAPV y a partir de ella se definen los instrumentos de ordenación territorial, tanto los planes integrales de ordenación del territorio a escala subregional denominados planes territoriales parciales (PTP) como los planes territoriales sectoriales (PTS), entre los que se encuentra el citado PTSL. Asimismo, el Decreto 90/2014 sobre protección, gestión y ordenación del paisaje en la ordenación del territorio del País Vasco establece una serie de instrumentos y mecanismos para integrar adecuados objetivos de calidad paisajística, y medidas a llevar a cabo al respecto, en los planes de ordenación territorial, con el fin de dar cumplimiento al Convenio Europeo del Paisaje del Consejo de Europa (2000).

Relacionada con la Ley de Ordenación del Territorio, la Ley 2/2006 del Suelo y Urbanismo del País Vasco no introduce normativa sustantiva alguna en materia de protec- 
ción del litoral. No obstante, afecta al mismo en tanto que permite que se dicten planes especiales de ordenación del litoral, siempre que no alteren la edificabilidad urbanística. Estos planes pueden establecer normas de protección adicional del litoral no dispuestas en el planeamiento general (Lazcano y Arzoz, 2009).

Por otro lado, la Ley 6/1998, de 13 de marzo, de Pesca Marítima tiene por objeto la ordenación de la actividad pesquera en aguas interiores, el marisqueo y los cultivos marinos mediante la protección y conservación de los recursos marinos, su aprovechamiento racional y el desarrollo ordenado de la actividad. Corresponde al Gobierno Vasco adoptar todas las medidas orientadas a la protección y conservación de dichos recursos. Por tanto, las zonas que sean declaradas áreas de reserva biológica, zonas vedadas, de arrecifes o zonas de especial protección se regirán por su norma reguladora.

Cabe deducir que la CAPV no dispone de una legislación específica para la GIZC. No se tienen en cuenta las singularidades del medio costero-marino y, cuando ello ocurre, la norma es sectorial y se vienen a proteger espacios por recursos específicos que contienen, como es el caso de la legislación pesquera.

\subsection{Competencias}

El hecho de que sobre el litoral se proyecten una gran variedad de usos y actividades dibuja un cuadro competencial en el que las potestades de unas y otras administraciones se entremezclan con frecuencia (Chica, 2008). Las funciones públicas relacionadas con la planificación y gestión costera están repartidas entre las tres escalas territoriales de Administración: la Administración General del Estado (AGE), la Administración autonómica y la Administración local.

El Estatuto de Autonomía del País Vasco, aprobado por la Ley Orgánica 3/1979, atribuye a la CAPV competencia exclusiva en las siguientes materias: bienes de dominio público y patrimoniales cuya titularidad corresponda a la Comunidad Autónoma, así como las servidumbres públicas en materias de sus competencias; pesca en aguas interiores, marisqueo y acuicultura; cofradías de pescadores, industria y navegación, sin perjuicio de la competencia del Estado en materia de comercio exterior; ordenación del territorio y del litoral, urbanismo y vivienda; transporte marítimo y puertos; y turismo. También es competencia de la CAPV el desarrollo y la ejecución de la legislación básica del Estado relacionada con el medio ambiente y ecología y la ordenación del sector pesquero. Por otro lado, le corresponde la ejecución de la legislación del Estado sobre puertos de interés general, cuando el Estado no se reserve su gestión directa, y sobre salvamento marítimo y vertidos industriales y contaminantes en las aguas territoriales del Estado correspondientes al litoral vasco. Por tanto, es la Administración autonómica la que tiene las mayores competencias en relación a sus áreas litorales, si bien algunas de ellas exigen una adecuada coordinación con la AGE. 
Es preciso tener en cuenta, no obstante, que la Ley 27/1983 de Territorios Históricos confiere a la CAPV una estructura administrativa muy compartimentada en la que las competencias se distribuyen entre las instituciones autonómicas (Gobierno y Parlamento Vasco) y los órganos forales de los Territorios Históricos (Diputaciones Forales y Juntas Generales). A los órganos forales de Bizkaia y Gipuzkoa se les atribuyen ciertas competencias que pueden incidir en la gestión del litoral, como el desarrollo y la ejecución de las normas emanadas de las Instituciones Comunes en materia de policía de aguas públicas continentales y de sus cauces naturales, riberas y servidumbres; o la ejecución dentro de su territorio de la legislación en materia de espacios naturales protegidos y la aprobación de los instrumentos de ordenación territorial y urbanística.

Como apuntan García-Sanabria et al. (2011), este reparto competencial es muy proclive a la conflictividad al no otorgar a los Territorios Históricos competencias sobre el medio ambiente como norma general. Por ejemplo, el modelo de gestión de la Reserva de la Biosfera de Urdaibai es diferente a la que la Ley de Territorios Históricos diseña en materia de espacios naturales protegidos ya que las decisiones normativas y ejecutivas más importantes que afectan a este espacio se adoptan por la Administración autonómica, en detrimento del papel de la Diputación Foral de Bizkaia (Lazcano y Arzoz, 2009).

\subsection{Instituciones}

El sector público precisa de unidades administrativas especializadas para ejecutar su política territorial. En el País Vasco coexisten diferentes órganos responsables de la gestión del litoral, si bien no hay una única institución, Administración o departamento específico para la GIZC. Esta situación es el reflejo del reparto competencial descrito anteriormente. No obstante, en algunos departamentos del Gobierno Vasco existen direcciones que tienen a su cargo cometidos muy específicos sobre el medio costero-marino.

El Departamento de Medio Ambiente, Planificación Territorial y Vivienda es el que más responsabilidad asume en cuanto a la gestión del litoral. Entre sus áreas de actuación destacan la ordenación del territorio y del litoral, el suelo y urbanismo, el medio ambiente, la ordenación de recursos naturales y conservación de la naturaleza, las aguas, los transportes terrestres y marítimos y los puertos.

La Viceconsejería de Planificación Territorial, tiene por objeto facilitar los servicios administrativos de carácter general, así como impulsar la planificación, el seguimiento y la evaluación de las actividades del Departamento en el marco de una planificación territorial estratégica. Esta institución ha sido responsable del PTSL, principal instrumento de gestión costera de la CAPV sobre el cual se hablará en el próximo apartado.

Por su parte, la Viceconsejería de Medio Ambiente se ocupa de la gestión de distintas autorizaciones de carácter medioambiental, así como de las afecciones ambien- 
tales de los diferentes entornos. Además, promueve la adopción de los instrumentos de protección del medio natural, la educación y la sensibilización ambiental dentro de un marco propio de planificación ambiental. Esta Viceconsejería gestiona la Sociedad Pública de Gestión Ambiental Ihobe, dedicada a la aplicación de la política ambiental y la extensión de la cultura de la sostenibilidad en la CAPV.

La Agencia Vasca del Agua URA es un ente público de derecho privado que también se encuentra vinculado con el Departamento de Medio Ambiente y Política Territorial. Esta institución tiene como objeto llevar a cabo la política del agua en Euskadi siendo algunas de sus grandes líneas de actuación: la mejora del estado ecológico de las masas de agua, la obtención del abastecimiento universal en cantidad y calidad suficiente y la culminación de los planes de saneamiento.

Otro de los Departamentos que ejerce funciones de administración de los recursos marino-costeros es el de Desarrollo Económico e Infraestructuras. En su seno destacan por su incidencia en el ámbito de estudio la Dirección de Desarrollo Rural y Litoral y Políticas Europeas, la Dirección de Pesca y Acuicultura la Corporación del Gobierno Vasco para el Desarrollo del Medio Rural y Marino, Fundación Hazi, y la Viceconsejería de Turismo y Comercio.

Corresponde a la Dirección de Desarrollo Rural y Litoral y Políticas Europeas, entre otras atribuciones, la promoción y formación agraria y náutico-pesquera, el fomento de la cultura emprendedora empresarial en los sectores de la agricultura, la pesca y la alimentación, y la elaboración y propuesta de programas de actuaciones con el fin de detener el declive de las zonas pesqueras. Mientras, la Fundación Hazi tiene como fin impulsar la competitividad y la sostenibilidad del sector primario y alimentario y el desarrollo sostenible del medio rural y litoral. Para ello presta un servicio integral en colaboración con los agentes e instituciones implicados en el mismo.

En cuanto a la Viceconsejería de Comercio y Turismo, entre otras funciones, coordina y controla los procedimientos de ordenación turística (registro, autorizaciones, reclamaciones e inspección) y promueve una gestión turística sostenible impulsando planes de dinamización turística y entes de cooperación de carácter comarcal.

Respecto a las instituciones de la AGE, la Dirección General de Sostenibilidad de la Costa y del Mar dispone de una Demarcación Periférica en Bizkaia, la Demarcación de Costas en el País Vasco. Entre sus funciones destacan las actuaciones e inversiones para la sostenibilidad de la costa (obras e infraestructuras orientadas a la gestión sostenible y regeneración del litoral), la aplicación de los instrumentos jurídicos para la protección del DPMT y la participación coordinada en la gestión territorial de la costa con otras administraciones (Gobierno de España, 2011). Así, pese a que se ha constatado una escasa colaboración entre las Administraciones estatal y autonómica (GarcíaSanabria et al., 2011), se han llevado a cabo importantes actuaciones de recuperación ambiental en el borde costero. 
Por tanto, fruto del reparto competencial, el litoral vasco se administra a través de las diferentes instituciones sectoriales cuya coordinación y cooperación es insuficiente para alcanzar una gestión integrada. Aún no se ha creado un órgano de coordinación interinstitucional que constituya un espacio de encuentro entre las tres escalas de la Administración. La institución con más competencias para la gestión integrada del litoral y que por tanto podría ejercer un liderazgo en su gestión es el Gobierno Vasco.

\subsection{Instrumentos}

Las normativas autonómicas mencionadas han dado lugar a una serie de planes y programas orientados a la preservación y uso ordenado de los recursos naturales. Sin embargo, ninguno de los principales instrumentos que afectan al litoral (Tabla 2) tiene una visión estratégica integrada.

En la CAPV, si bien hasta el momento se han realizado algunos trabajos relacionados con la protección y conservación del medio marino al amparo de la Directiva Europea Marco de Agua y la Directiva Marco de la Estrategia Marina Europea, no existen estudios que traten de forma integrada la gestión costera. La única referencia al tema en cuestión es un breve trabajo realizado por la Red Española de Gestión Integrada de Áreas Litorales (García-Sanabria et al., 2011) con la pretensión de crear un espacio de encuentro para fomentar la coordinación y cooperación entre los diferentes agentes públicos y privados del litoral español. El citado documento describe el estado de la gestión pública de las costas de cada Comunidad Autónoma, entre ellas la vasca.

Otros documentos relacionados no cuentan con un enfoque estratégico. Los instrumentos con mayor carácter integrador para la gestión del litoral son los de ordenación territorial. Las Directrices de Ordenación Territorial (DOT), cuya revisión se encuentra en fase aprobación inicial (Gobierno Vasco, 2018a), constituyen el marco general de referencia de los restantes instrumentos. A pesar de que dedican escasas determinaciones para el espacio costero-marino, por un lado, se introduce alguna previsión en un breve apartado de tratamiento del espacio litoral y medio marino (Gobierno Vasco, 1997). Se les dota de categoría de especial protección a las rías, estuarios, playas y acantilados costeros. Respecto al urbanismo, se establecen límites máximos a la oferta del suelo para segunda residencia, gran parte del cual se ubica en zonas costeras.

El desarrollo de las directrices mencionadas se articula a través de los PTP y los PTS. Los PTP concretan las determinaciones de las DOT en las quince áreas funcionales de escala subregional, de las cuales seis pertenecen al ámbito de estudio, todas ellas con su PTP aprobado (Tabla 2). No obstante, como señalan Lazcano y Arzoz (2009), a pesar de que estos instrumentos pueden establecer determinaciones complementarias de protección de la costa, las referencias a este tipo de mecanismos son generalmente escasas. 
Tabla 2. Principales instrumentos que afectan a la gestión de las zonas costeras de la CAPV

\begin{tabular}{|c|c|}
\hline Recurso/actividad regulada & Instrumento (año de aprobación) \\
\hline \multirow{10}{*}{$\begin{array}{l}\text { Ordenación del territorio y } \\
\text { del litoral, suelo y urbanismo }\end{array}$} & Directrices de Ordenación del Territorio (DOT) (1997) \\
\hline & PTP de Bilbao Metropolitano (2006) \\
\hline & PTP de Mungia (2016) \\
\hline & PTP de Gernika-Markina (2016) \\
\hline & PTP de Eibar (Bajo Deba) (2005) \\
\hline & PTP de Zarautz-Azpeitia (Urola Costa) (2006) \\
\hline & $\begin{array}{l}\text { PTP de Donostia-San Sebastián (Donostialdea-Bajo } \\
\text { Bidasoa) (2016) }\end{array}$ \\
\hline & PTS de Protección y Ordenación del Litoral (2007) \\
\hline & PTS de los Márgenes de Ríos y Arroyos (1999) \\
\hline & PTS de Zonas Húmedas (2001) \\
\hline \multirow[t]{4}{*}{ Paisaje } & $\begin{array}{l}\text { Catálogo de Paisaje de Donostia-San Sebastián } \\
\text { (Donostialdea-Bajo Bidasoa) (2016) }\end{array}$ \\
\hline & $\begin{array}{l}\text { Catálogo de Paisaje de Zarautz-Azpeitia } \\
\text { (Urola Costa) (2012) }\end{array}$ \\
\hline & $\begin{array}{l}\text { Determaciones de Paisaje de Zarautz-Azpeitia } \\
\text { (Urola Costa), las cuales han sido integradas en } \\
\text { el PTP correspondiente en } 2018\end{array}$ \\
\hline & $\begin{array}{l}\text { Planes de Acción del Paisaje de Muskiz (2014), Zierbena } \\
\text { (2016), Getxo (2015), Barrika (2014), Bermeo (2017), } \\
\text { Lekeitio (2015), Deba (2016), Getaria (2014), } \\
\text { Donostia-San Sebastián (2015) y Pasaia (2017) }\end{array}$ \\
\hline \multirow[t]{6}{*}{ Conservación de la naturaleza } & PORN del tramo litoral Deba-Zumaia (2009) \\
\hline & PRUG de la Reserva de la Biosfera de Urdaibai (2016) \\
\hline & Plan Especial del Biotopo Protegido de Iñurritza (2006) \\
\hline & $\begin{array}{l}\text { Plan Especial de Protección y Ordenación de los } \\
\text { Recursos Naturales del Área de Txingudi (1994) }\end{array}$ \\
\hline & $\begin{array}{l}\text { Planes de Gestión de los espacios Natura } 2000 \text { costeros } \\
\text { (2012 a 2014) }\end{array}$ \\
\hline & $\begin{array}{l}\text { Estrategias de Cambio Climático (2015), Geodiversidad } \\
\text { (2014) y Biodiversidad (2016) }\end{array}$ \\
\hline Pesca & Plan Estratégico de la Pesca y Acuicultura Euskadi (2014) \\
\hline Turismo & Plan Director de Turismo de la Costa Vasca (2016) \\
\hline
\end{tabular}

Fuente: Elaboración propia. 
Los PTS son planes elaborados por los distintos Departamentos del Gobierno Vasco que tienen competencias de incidencia territorial. El PTS de Protección y Ordenación del Litoral (PTSL) (Gobierno Vasco, 2007) constituye el marco planificador más completo de la zona costero-marina vasca. Su objetivo es aplicar criterios de protección, mejora y conservación en los siguientes tres espacios: el medio terrestre, que coincide con la Zona de Influencia definida en la Ley de Costas; los medios de transición, que abarca el espacio marítimo-terrestre (zona intermareal comprendida entre la línea de bajamar escorada o máxima viva equinoccial y la línea de pleamar máxima viva equinoccial) y las rías (se extienden desde su desembocadura por las márgenes de los ríos hasta la cota de $5 \mathrm{~m}$ sobre el nivel del mar); y el medio marino, que comprende el espacio entre la línea de bajamar escorada o máxima viva equinoccial y la isobata de $50 \mathrm{~m}$ (Gobierno Vasco, 2007).

En el caso del medio terrestre se zonifica el territorio en sectores homogéneos a través de categorías de ordenación, definidas en función de la capacidad de acogida y la valoración ambiental (especial protección, mejora ambiental, forestal, agroganadera y campiña y uso especial), y de condicionantes superpuestos, que limitan el desarrollo de ciertas actividades según el tipo de riesgo considerado (áreas vulnerables a la contaminación de acuíferos, áreas erosionables y áreas inundables). Una vez establecida esta zonificación se fija el régimen de usos (propiciados, admisibles, prohibidos e improcedentes) de cada sector para cada actividad (Gobierno Vasco, 2007).

Respecto a los medios de transición, su ordenación se basa en la coordinación con el PTS de Ordenación de los Márgenes de Ríos y Arroyos (Gobierno Vasco, 1999). Este plan, por un lado, establece la normativa general en materia de aguas y, por otro, divide todos los cursos de agua por tramos de problemática homogénea a partir del análisis de tres componentes: ambiental, hidráulica y urbanística.

En el medio marino, el PTSL define sectores de planificación (zonas de conservación y mejora ambiental, zonas de ocio y esparcimiento, zonas de explotación de recursos primarios y zonas de localización de infraestructuras y vertidos) y directrices orientativas de los usos a desarrollar en los mismos, identificando usos preferentes y excluyendo otros en algunos de los sectores de planificación. Además, incorpora determinaciones específicas para la regulación de los vertidos al dominio público (Gobierno Vasco, 2007).

Quedan fuera del ámbito de aplicación de este PTSL otros elementos cuya ordenación depende de otros instrumentos como las infraestructuras portuarias, los espacios naturales protegidos y algunas zonas húmedas. Éstas últimas quedan reguladas por el PTS de Zonas Húmedas (Gobierno Vasco, 2004).

La revisión de las DOT (Gobierno Vasco, 2018a) recoge además la habilitación e integración en la planificación territorial de la denominada senda del mar, que concibe 
como un itinerario de movilidad no motorizada para el disfrute del litoral que recorre todo el borde costero. Asimismo, la revisión de las DOT insta a integrar en la planificación territorial al Camino de Santiago (Gobierno Vasco, 2018a), cuya ruta norte discurre por la costa guipuzcoana (Porcal-Gonzalo et al., 2012).

Asimismo desde 2014 se están impulsando una serie instrumentos de gestión del paisaje para integrar los objetivos del Convenio Europeo del Paisaje en la ordenación territorial del País Vasco. Se trata de Catálogos del Paisaje y Determinaciones del Paisaje a escala subregional (que se redactan para que sus determinaciones sean integradas en los planes territorial parciales, como ha sido el caso del PTP de Urola Costa en 2018) y de Planes de Acción del Paisaje a escala local.

Otros instrumentos relacionados con la gestión del litoral son los derivados de los distintos sectores de actividad estrechamente vinculados con este ámbito: la pesca, la navegación y el turismo. En este sentido, el Plan Estratégico de la Pesca y Acuicultura Euskadi 2020 (Gobierno Vasco, 2016b) plantea la revalorización de sus productos y diversificación económica, así como el fomento de la sostenibilidad ambiental costera y la cooperación transfronteriza estatal e internacional. Por otro lado, el Plan Director de Turismo de la Costa Vasca (Basquetour, 2016), es una herramienta que apuesta por la gestión turística sostenible en comarcas como la Costa Vasca. En esta línea, la Estrategia de Geodiversidad de la CAPV 2020 (Gobierno Vasco, 2014) persigue fomentar la conservación, la educación, y el uso sostenible de la geodiversidad, así como el geoturismo en torno a los lugares de interés geoturístico presentes en la costa vasca. Por su parte, la Estrategia vasca de cambio climático 2050 tiene entre sus objetivos el de integrar la variable del cambio climático en la gestión costera, dada la vulnerabilidad del litoral ante procesos como el ascenso del nivel del mar o el aumento de episodios de oleaje extremo (Gobierno Vasco, 2015).

\subsection{Formación y capacitación}

Como confirman Barragán et al. (2008) los documentos internacionales sobre GIZC insisten en la importancia de la figura del gestor costero que, dentro del contexto administrativo considerado, puede identificarse con un funcionario público o con un técnico contratado al servicio de la Administración competente en costas. Por tanto, la formación y capacitación de este tipo de personas es fundamental para abordar con éxito la GIZC. La formación especializada en este campo se consigue adquiriendo conocimientos científicos socio-ambientales, así como habilidades para la gestión de políticas públicas (planificación estratégica, resolución de conflictos, técnicas de negociación, técnicas para la toma de decisiones, etc.).

La Administración vasca no oferta cursos de capacitación con contenidos formativos relacionados con la GIZC y los cursos existentes son de carácter operativo, orien- 
tados a mejorar la gestión diaria (García-Sanabria et al., 2011). Actualmente los perfiles profesionales de los administradores o gestores costeros vascos siguen estando muy vinculados a la ingeniería y no se ha llegado a valorar suficientemente la necesidad de formación en materia de habilidades sociales y destrezas personales.

En relación a la formación universitaria, por un lado, existen dos titulaciones de la Universidad del País Vasco (UPV/EHU) con rango de Grado, la de Geografía y Ordenación del Territorio y la de Ciencias Ambientales, que aportan una visión interdisciplinar y global de la problemática territorial lo cual resulta imprescindible para enfrentarse a los asuntos costeros. De esta forma, estos grados capacitan para reconocer, analizar y tratar las interrelaciones del sistema físico-natural con el económico, social y cultural.

Por otro lado, en la misma universidad se imparten varios posgrados oficiales que pueden responder a alguna de las necesidades específicas de la GIZC. El Máster Universitario en Contaminación y Toxicología Ambientales proporciona una educación científica experimental muy orientada a la investigación y entre sus líneas de especialización se encuentra la contaminación marina. El Erasmus Mundus Master of Science in Marine Environment and Resources (MER) responde a los retos de la nueva Directiva Marco sobre la Estrategia Marina y la Directiva Marco de Agua, incluyendo formación sobre la GIZC entre sus temas. No obstante, el objetivo del programa va más orientado a que el estudiante adquiera destrezas científico-técnicas que habilidades personales para la gestión. Por su parte, el Máster Universitario en Gobernanza y Estudios Políticos permite profundizar en temas relacionados con el comportamiento y análisis político, administración y políticas públicas, participación política y gobernanza.

A nivel de ciclos formativos, el Centro Integral para la Formación, Inserción y Desarrollo Rural-Litoral de la CAPV cuenta con varios centros distribuidos en los tres territorios históricos con una oferta formativa basada en la impartición de Grados Medios y Grados Superiores, así como certificados de especialidad y títulos profesionales homologados internacionalmente. Entre ellos se encuentran la Escuela Marítimo Pesquera de Pasaia y la de Bermeo y otro centro colaborador en Ondarroa especializados en la formación marítimo-pesquera. Su misión es contribuir de manera proactiva a la mejora de la competitividad del sector náutico-pesquero y al fomento del desarrollo rural y litoral.

Así, la Administración pública vasca no contempla programas para dotar a los administradores de conocimientos en GIZC. Sus perfiles curriculares muestran una alta especialización técnica y operativa en perjuicio de las habilidades y visiones estratégicas. Lo mismo ocurre con la formación universitaria y de ciclos formativos. Más allá del MER no se imparte formación con un temario relacionado con la GIZC. 


\subsection{Conocimiento e información}

En el marco territorial del litoral de la CAPV se dispone de un conocimiento alto de los subsistemas físico-natural y socioeconómico, siendo bien conocidos, por lo general, los principales problemas y sus causas. Sin embargo, esta información, si bien es accesible a través de Internet, está dispersa y no existe un único organismo encargado de su recopilación, ordenamiento y posterior análisis. Entre la información más relevante destacan la cartografía recogida en la Infraestructura de Datos Espaciales de Euskadi, los informes de seguimiento y evaluación, los datos y estudios estadísticos, los artículos de investigación, así como los textos normativos y de planificación.

En cuanto a la generación del conocimiento, por una parte, destaca el papel de la Unidad de Investigación Marina de AZTI-Tecnalia, centro tecnológico de investigación marina y alimentaria. Su misión es aportar conocimientos científicos sobre el funcionamiento de los ambientes costeros, necesarios para lograr una gestión sostenible de los recursos, bienes y servicios. El Patronato de la Fundación AZTI está formado por representantes de empresas e instituciones del sector pesquero, alimentario y medio ambiente marino, así como del Gobierno Vasco. Entre las líneas de investigación de esta unidad se encuentra la de GIZC y estudia la calidad ambiental de la zona litoral, el desarrollo de métodos de evaluación del impacto ambiental, la protección y recuperación del medio marino y estuárico, la gestión sostenible de los recursos naturales y el asesoramiento a las administraciones públicas para el establecimiento de planes de gestión de la actividad costera (planes de dragados, ordenación de la actividad acuícola, etc.).

Por otro lado, la UPV/EHU cuenta también con diversos departamentos y grupos de investigación que centran sus estudios en el medio marino-costero. Parte de este trabajo es desarrollado en la Estación Marina de Plentzia, centro de investigación y docencia que tiene como objetivo promover la investigación sobre la salud del ecosistema marino y los efectos de las alteraciones medioambientales sobre la salud humana. Los estudios que aquí se realizan incluyen el análisis tanto de especies animales de interés comercial (pesquerías, marisqueo y acuicultura) como silvestres, para la evaluación de la salud del medio marino.

En conclusión, puede considerarse que, tanto por parte de la Administración como por parte de las instituciones generadoras de conocimiento, existe una amplia información sobre el medio costero-marino vasco. El problema es que no ha sido integrada y analizada convenientemente, con lo que no se llega a alcanzar un conocimiento integrado del medio ni un enfoque omnicomprensivo de los problemas costeros (GarcíaSanabria et al., 2011). 


\subsection{Educación y concienciación}

En cuanto a iniciativas de educación para los ciudadanos y usuarios del litoral en general relacionados con la sostenibilidad de la costa y el medio marino, el Gobierno Vasco ha desarrollado una estructura de educación ambiental a través de diferentes iniciativas y programas entre los cuales destaca un proyecto orientado al medio costero-marino.

Los centros Ingurugela son una red de equipamientos públicos de apoyo al profesorado y a los centros escolares que coordinan planes y programas de educación ambiental en el sistema educativo no universitario. Esta red está formada por cinco centros y 14 profesores/as cuyo trabajo anual se organiza tomando como base el Programa de Educación Ambiental suscrito y aprobado por los Departamentos de Educación, Universidades e Investigación y Medio Ambiente y Ordenación del Territorio. Este programa estableció tres líneas de trabajo que siguen vigentes en la actualidad: investigación y experimentación (a través de Agendas 21 Escolares), formación del profesorado (mediante los cursos de GARATU) y sensibilización (a través del programa Aztertu, los Encuentros de Educación Ambiental y los Centros Ambientales). El programa Aztertu recoge dos campañas de sensibilización, sobre el estado medioambiental de la costa (Azterkosta) y de los ríos (Ibaialde), dirigidas a centros escolares y a la ciudadanía. Ingurugela junto con el Servicio de Formación Ambiental son quienes coordinan el diseño y la elaboración de los materiales de dichas campañas.

Por otro lado, Azterkosta (Gobierno Vasco, 2018c) es la adaptación para la CAPV del programa europeo de Educación Ambiental sobre el litoral Coastwatch. Se trata de un instrumento de ayuda para conocer mejor las peculiaridades del litoral, difundir su problemática y ampliar la concienciación social sobre la necesidad de proteger los espacios naturales costeros. Para ello cada año se realiza una difusión del programa entre diferentes colectivos, como centros escolares, de tiempo libre, asociaciones ecologistas, ayuntamientos, etc., proponiendo su participación. También se ofrece la oportunidad de realizar valoraciones generales sobre el estado de la costa vasca y de aportar opiniones e ideas para mejorar la gestión de la zona costera, detectando problemas, denunciando actuaciones ilegales o proponiendo áreas cuya conservación sería interesante lograr.

A pesar de los esfuerzos del Gobierno Vasco en materia de educación ambiental, no se ha encontrado ninguna referencia a la GIZC. La sociedad toma parte en las actividades voluntarias propuestas por los programas de sensibilización, pero no reconoce la pertinencia de la gestión integrada costero-marina para lograr la sostenibilidad de estos espacios. 


\subsection{Participación}

El hecho de que gran parte del espacio litoral forme parte del dominio público hace indispensable la participación ciudadana en la gestión costera. Además, en este ámbito coinciden diversos intereses y actores que con frecuencia entran en conflicto como consecuencia de las decisiones tomadas por la Administración en relación con la concesión de autorizaciones y prohibiciones de determinados usos. En este sentido, la gestión participativa constituye un elemento imprescindible en la búsqueda del consenso (Barragán et al., 2008; Farinós, 2011; García-Sanabria, 2015). En el proceso de toma de decisiones participan distintos órganos encargados de asesorar o fomentar la cooperación y coordinación entre los diferentes agentes sociales e institucionales, públicos y privados, con intereses en el litoral. En el País Vasco no existe aún un órgano de participación específico para la GIZC, pero sí otros que se comentan a continuación.

Por un lado, el Gobierno Vasco cuenta con una serie de órganos, entre estos destacan por su mayor relación con el litoral el Comité Consultivo de Pesca, el Patronato y el Consejo de Cooperación de la Reserva de la Biosfera de Urdaibai y los Consejos Asesores y el Órgano Consultivo de Puertos y Asuntos Marítimos Vascos.

El Comité Consultivo de Pesca tiene como objetivo encauzar la participación y consulta de los sectores sociales y actividades relacionadas con la pesca. Entre sus funciones destacan las siguientes: deliberar sobre los asuntos que le someta el Presidente, asesorándole al respecto; elaborar los informes que le sean solicitados sobre cuestiones que afecten al sector pesquero; y elevar propuestas y hacer recomendaciones en materia pesquera a los organismos, administraciones y agentes sociales que correspondan. Este comité está formado por personas pertenecientes al departamento competente en materia de pesca y una larga lista de federaciones y asociaciones relacionados con esta actividad.

El Patronato de la Reserva de la Biosfera de Urdaibai es un órgano de coordinación interadministrativa y de participación, lugar de encuentro y coordinación y garante de la coherencia de las políticas sectoriales. Está compuesto por los representantes de las administraciones que tienen competencias en Urdaibai (Estado, Gobierno Vasco, Diputación de Bizkaia y Ayuntamientos) y por organismos, asociaciones y sindicatos del entorno. Su función es la de emitir informes preceptivos sobre las actuaciones que se pretenden realizar en áreas de especial protección y sobre los planes con incidencia en el medio físico. Por su parte, el Consejo de Cooperación de la Reserva de la Biosfera de Urdaibai es un órgano colaborador del Patronato cuyo presidente traslada las iniciativas de este órgano al Pleno del Patronato.

Los Consejos Asesores de Puertos, concebidos como órganos de asesoramiento, consulta y debate de las actuaciones a desarrollar en cada puerto de titularidad autonómica, se circunscriben a los cuatro que, en función de su dimensionamiento, flota, capturas e incidencias, son los más importantes del litoral vasco (Bermeo, Ondarroa, Getaria y 
Hondarribia). En estos Consejos están representados la Administración autonómica, el Ayuntamiento y los colectivos vinculados con la pesca. Por otro lado, el Órgano Consultivo de Puertos y Asuntos Marítimos Vascos extiende su actividad a la totalidad de los puertos transferidos a la Administración de la CAPV afectando a las competencias en materia de transporte marítimo y fluvial, salvamento marítimo, etc. Entre sus funciones están las de analizar, estudiar, debatir e informar los asuntos, planes y proyectos de disposiciones normativas que elabore y le traslade el Departamento competente en materia de puertos y asuntos marítimos, así como la de elevar a dicho Departamento opiniones y sugerencias referidas a la problemática que se suscite en torno a la actividad y vida portuaria y las infraestructuras de puertos. Este órgano consultivo está compuesto por representantes de la Administración autonómica y local (Diputaciones de Bizkaia y Gipuzkoa) y los sectores implicados entre los que están las cofradías de pescadores, los clubes náuticos, los sindicatos, las autoridades portuarias, etc.

Otro órgano consultivo que puede jugar un papel importante en la gestión costera es el grupo de acción local del sector pesquero, que gestiona a proyectos para el desarrollo sostenible de las zonas de pesca estimulando la movilización de los recursos locales para el proceso de desarrollo y la capacidad organizativa de estas zonas a través de la gobernanza (reuniendo y aunando voluntades y esfuerzos de organizaciones y agentes públicos y privados implicados).

Por otro lado, el Gobierno Vasco ha desarrollado una iniciativa de gobernanza a través de la transparencia, participación y colaboración. Se trata de la plataforma Irekia, un canal de comunicación directa entre la ciudadanía y la Administración a través de Internet.

\section{Conclusiones}

Se concluye que en la CAPV no hay una orientación estratégica para gestionar la costa desde un enfoque integrado. La gestión costera se lleva a cabo desde distintas políticas sectoriales y existen múltiples marcos reguladores con influencia en la toma de decisiones. Las responsabilidades en relación con el litoral están repartidas entre las distintas escalas administrativas y, a su vez, entre distintos entes sectoriales. El complejo marco competencial no facilita la gestión coordinada y puede desencadenar conflictos entre distintos niveles administrativos. Si bien el conocimiento del medio costero y sus problemáticas es elevado y se dedican significativos esfuerzos en formación, educación y participación, la integración social en la toma de decisiones referidas al litoral es insuficiente para desarrollar un modelo de GIZC. Se sintetizan las conclusiones del diagnóstico en la siguiente matriz DAFO (Tabla 3). 
Tabla 3. Análisis DAFO de la gestión del litoral vasco

\begin{tabular}{|c|c|}
\hline DEBILIDADES & FORTALEZAS \\
\hline $\begin{array}{l}\text { La gestión pública del litoral se rige } \\
\text { por criterios sectoriales. }\end{array}$ & $\begin{array}{l}\text { Solidez en la implantación de iniciativas } \\
\text { políticas en el País Vasco. }\end{array}$ \\
\hline $\begin{array}{l}\text { Ausencia de un marco regulador común } \\
\text { que integre la normativa sectorial. }\end{array}$ & $\begin{array}{l}\text { Integrador y estricto reglamento en materia } \\
\text { de Ordenación del Territorio. }\end{array}$ \\
\hline $\begin{array}{l}\text { Elevada fragmentación de las } \\
\text { responsabilidades relacionadas con la } \\
\text { gestión costera entre las diferentes } \\
\text { escalas administrativas. }\end{array}$ & $\begin{array}{l}\text { Firma de acuerdos competenciales entre las } \\
\text { escalas local y autonómica para la gestión } \\
\text { de espacios naturales. }\end{array}$ \\
\hline $\begin{array}{l}\text { Falta de mecanismos de coordinación } \\
\text { entre las instituciones que ejecutan la } \\
\text { política territorial del litoral. }\end{array}$ & $\begin{array}{l}\text { El Departamento de Medio Ambiente y } \\
\text { Política Territorial tiene en cuenta la } \\
\text { planificación del litoral (PTSL) de forma } \\
\text { singularizada. }\end{array}$ \\
\hline $\begin{array}{l}\text { No se ha desarrollado ningún instrumento } \\
\text { estratégico específico para la GIZC. }\end{array}$ & $\begin{array}{l}\text { Existe un instrumento de planificación } \\
\text { territorial muy completo que considera } \\
3 \text { espacios litorales: el medio terrestre, } \\
\text { las zonas de transición y el medio marino. }\end{array}$ \\
\hline $\begin{array}{l}\text { Formación muy técnica de los funcionarios } \\
\text { y técnicos de la Administración olvidando } \\
\text { las habilidades personales para la gestión } \\
\text { de políticas públicas. }\end{array}$ & $\begin{array}{l}\text { Comienzan a aparecer ofertas formativas } \\
\text { que pueden capacitar y motivar a los } \\
\text { profesionales para liderar iniciativas } \\
\text { de GIZC. }\end{array}$ \\
\hline $\begin{array}{l}\text { La información referida al medio } \\
\text { costero-marino vasco está dispersa. }\end{array}$ & $\begin{array}{l}\text { Elevada cantidad, calidad y accesibilidad } \\
\text { de la información. }\end{array}$ \\
\hline $\begin{array}{l}\text { Los programas de educación no tienen el } \\
\text { objetivo de trasladar el sentido de } \\
\text { pertinencia de la GIZC para lograr la } \\
\text { sostenibilidad del ámbito de estudio. }\end{array}$ & $\begin{array}{l}\text { Buena acogida y trayectoria del programa } \\
\text { de sensibilización Azterkosta en los } \\
\text { territorios costeros año tras año. }\end{array}$ \\
\hline $\begin{array}{l}\text { No existe un órgano de participación } \\
\text { diferenciado para la GIZC. }\end{array}$ & $\begin{array}{l}\text { Participación pública y cooperación } \\
\text { administrativa institucionalizadas. }\end{array}$ \\
\hline AMENAZAS & OPORTUNIDADES \\
\hline $\begin{array}{l}\text { Modelo reactivo de gestión pública } \\
\text { incompatible con el dinamismo de los } \\
\text { problemas socio-ambientales. }\end{array}$ & $\begin{array}{l}\text { La necesidad que existe de integrar el } \\
\text { cambio climático en la gestión costera } \\
\text { puede favorecer la creación de políticas } \\
\text { de GIZC. }\end{array}$ \\
\hline $\begin{array}{l}\text { Continuidad de la interpretación sectorial } \\
\text { de la normativa aplicable. }\end{array}$ & $\begin{array}{l}\text { Posibilidad del Gobierno Vasco para } \\
\text { negociar con la AGE en materia de } \\
\text { competencias a través de las Comisiones } \\
\text { Bilaterales. }\end{array}$ \\
\hline
\end{tabular}


Tabla 3. Análisis DAFO de la gestión del litoral vasco (continuación)

\begin{tabular}{|l|l|}
\hline AMENAZAS & OPORTUNIDADES \\
\hline $\begin{array}{l}\text { Desencuentro institucional entre la AGE } \\
\text { y la Administración autonómica. }\end{array}$ & $\begin{array}{l}\text { Capacidad del Gobierno Vasco de liderar, } \\
\text { con el resto de instituciones, acciones } \\
\text { coordinadas en el ámbito de estudio. }\end{array}$ \\
\hline $\begin{array}{l}\text { Incumplimiento de los mecanismos } \\
\text { dispuestos para la planificación y gestión } \\
\text { del litoral por parte de las Administraciones } \\
\text { locales. }\end{array}$ & $\begin{array}{l}\text { Preocupación por coordinar todos los } \\
\text { instrumentos de planificación y gestión de } \\
\text { zonas costeras (los PTP, PTS, Programa } \\
\text { de Desarrollo Litoral, etc.). }\end{array}$ \\
\hline $\begin{array}{l}\text { Mantenimiento de la visión sectorial de los } \\
\text { programas docentes especializados } \\
\text { en temas marino-costeros. }\end{array}$ & $\begin{array}{l}\text { Aumento progresivo del conocimiento del } \\
\text { litoral vasco que podría facilitar a la } \\
\text { Administración emprender acciones con } \\
\text { mayor solvencia. }\end{array}$ \\
\hline $\begin{array}{l}\text { Desequilibrio de la generación de } \\
\text { conocimiento científico más volcado } \\
\text { a temas marinos que a costeros. }\end{array}$ & $\begin{array}{l}\text { Consolidación de los programas educativos } \\
\text { dirigidos a todos los públicos que posibiliten } \\
\text { la comprensión de los procesos } \\
\text { socio-ambientales del litoral. }\end{array}$ \\
\hline $\begin{array}{l}\text { Restricciones de acceso a los órganos } \\
\text { de participación para algunas } \\
\text { Administraciones (Estado y Ayuntamientos). }\end{array}$ & $\begin{array}{l}\text { Aprovechamiento de los canales de } \\
\text { comunicación directa entre la ciudadanía y } \\
\text { la Administración para la gestión costera. }\end{array}$ \\
\hline
\end{tabular}

Fuente: Elaboración propia.

La situación actual presenta a su vez ciertas fortalezas y oportunidades que pueden aprovecharse para desarrollar en el futuro un modelo de GIZC en la CAPV. Está aumentando la concienciación sobre la problemática socio-ambiental del litoral vasco. Frente a la vulnerabilidad de la costa ante procesos relevantes como el aumento del nivel del mar, el Gobierno Vasco (2015) reconoce la necesidad integrar el cambio climático en la gestión costera. Este complejo reto, que requiere de la implicación y coordinación del conjunto de las políticas sectoriales, debe aprovecharse para impulsar el desarrollo de planes formativos y de políticas específicas sobre GIZC.

Son necesarias investigaciones futuras para realizar un diagnóstico comparado de los marcos de gestión del litoral de las distintas Comunidades Autónomas y de otros países con territorios costeros, con el fin de identificar buenas prácticas y de progresar hacia el establecimiento de políticas adecuadas de GIZC. 


\section{Bibliografía}

Ahlhorn, F., 2018. Integrated coastal zone management: Status, challenges and prospects. Wiesbaden: Springer Vieweg. DOI: https://doi.org/10.1007/978-3-658-17052-3

Arto, I., 2010. La política ambiental vasca: pasado, presente y futuro. Ekonomiaz, 25, $442-470$.

Barragán, J.M., 2004. Las áreas litorales de España: del análisis geográfico a la gestión integrada. Barcelona: Ariel.

Barragán, J.M., Chicha, J.A. y Pérez, M., 2008. Propuesta de Estrategia Andaluza de Gestión Integrada de Zonas Costeras. Sevilla: Consejería de Medio Ambiente de la Junta de Andalucía.

Barragán, J.M., 2010. La gestión de los espacios y recursos costeros en España: Política e instituciones de una legislatura (2004-2008). Estudios Geográficos 268, 39-65.

DOI: https://doi.org/10.3989/estgeogr.0450

Barragán, J.M. y de Andrés, M., 2016. Aspectos básicos para una gestión integrada de las áreas litorales de España: conceptos, terminología, contexto y criterios de delimitación. Journal of Integrated Coastal Zone Management, 16, 171-183. DOI: https://doi.org/10.5894/rgci638

Borja, A., Bald, J., Franco, J., Larreta, J. et al., 2018. Red de seguimiento del estado ecológico de las aguas de transición y costeras de la CAPV. Informe de resultados. Campaña 2017. Vitoria-Gasteiz: Gobierno Vasco.

Chica, J.A., 2008. Conservación y desarrollo en el litoral español y andaluz: planificación y gestión de espacios naturales protegidos. Madrid: CEP Editorial.

Chust, G., Borja, Á., Caballero, A., Irigoien X. et al., 2011. Climate change impacts on coastal and pelagic environments in the southeastern Bay of Biscay. Climate Research, 48, 307-332. DOI: https://doi.org/10.3354/cr00914

Cicin-Sain, B., 1993. Sustainable development and integrated coastal management. Ocean and Coastal Management, 21, 11-43. DOI: https://doi.org/10.1016/0964-5691(93)90019-U

Clark, J.R., 1995. Coastal zone management handbook. Boca Raton: CRC Press. DOI: https://doi.org/10.1201/9781315139654

Consejo de Europa (2000). Convenio Europeo del Paisaje. Florencia: Consejo de Europa.

Comisión Europea, 2013. Propuesta de Directiva del Parlamento Europeo y del Consejo por la que se establece un marco para la ordenación del espacio marítimo y la gestión integrada de las costas. COM(2013) 133 final. Bruselas: Comisión Europea.

Farinós, D., 2011. La gestión integrada de zonas costeras, ¿algo más que una ordenación litoral revisada? La GIZC como evolución de las prácticas de planificación y gobernanza territoriales. Valencia: Universitat de Valencia.

Farinós, J., García-Jiménez, M.J. y Aldrey, J.A., 2018. Desarrollo legislativo y planificador en materia territorial y urbanística a nivel español. En: Farinós, J. (ed) Territorio y Estados: Elementos para la coordinación de las políticas de ordenación del territorio en el siglo XXI. Valencia: Tirant Humanidades, pp. 959-1060.

Galparsoro, I., Liria, P., Legorburu, I., Bald, J., Chust, G., et al., 2012. A Marine Spatial Planning Approach to Select Suitable Areas for Installing Wave Energy Converters (WECs), on the Basque Continental Shelf (Bay of Biscay), Coastal Management, 40, 1-19. 
García-Sanabria, J., García-Onetti, J. y Barragán, J.M., 2011. Las Comunidades Autónomas y la Gestión Integrada de las Áreas Litorales de España. Materiales para un debate sobre gobernanza. Cádiz: Universidad de Cádiz.

García-Sanabria, J., 2015. Integrated Coastal Zone Management: A public policy. The need for communication, coordination and participation. Journal of Coastal Zone Management, 18: e113. DOI: https://doi.org/10.4172/2473-3350.1000e113

Gaztelumendi, S., Egaña, J., Liria, P., y Aranda, J.A. 2018. Description of a coastal impact event in Basque Country: the 9 February 2016 case. Advances in Science and Research, 15, 137-143. DOI: https://doi.org/10.5194/asr-15-137-2018

Gobierno de España, 2006. Gestión Integrada de las Zonas Costeras en España. Informe de España en cumplimiento de los requerimientos del capitulo VI de la Recomendación del Parlamento Europeo y del Consejo de 30 de Mayo de 2002 sobre la aplicación de la gestión integrada de las zonas costeras en Europa. Madrid: Ministerio de Medio Ambiente.

Gobierno de España, 2011. Objetivos, actuaciones y proyectos: Demarcación de Costas del País Vasco. Madrid: Ministerio de de Medio Ambiente, Medio Rural y Marino.

Gobierno Vasco, 1997. Directrices de Ordenación Territorial de la CAPV. BOPV29, de 12 de febrero de 1997.

Gobierno Vasco, 1999. Plan Territorial Sectorial de Ordenación de Márgenes de los Ríos y Arroyos de la CAPV. BOPV 34, de 18 de febrero de 1999.

Gobierno Vasco, 2004. Plan Territorial Sectorial de Zonas Húmedas de la CAPV. BOPV 222, de 19 de noviembre de 2004 .

Gobierno Vasco, 2007. Plan Territorial Sectorial de Protección y Ordenación del Litoral de la CAPV. BOPV 65, de 2 de abril de 2007.

Gobierno Vasco, 2014. Estrategia de Geodiversidad de la CAPV 2020. Bilbao: Ihobe.

Gobierno Vasco, 2015. Estrategia de Cambio Climático 2050 del País Vasco. Vitoria-Gasteiz: Servicio Central de Publicaciones del Gobierno Vasco.

Gobierno Vasco, 2016a. Plan Estratégico de la Pesca y Acuicultura Euskadi 2020. Vitoria-Gasteiz: Departamento de Desarrollo Económico y Competitividad del Gobierno Vasco.

Gobierno Vasco, 2016b. Plan Director de Turismo de la Costa Vasca. Vitoria-Gasteiz: Dirección de Turismo del Gobierno Vasco.

Gobierno Vasco, 2016c. Estrategia de Biodiversidad del País Vasco 2030 y Primer Plan de Acción 2020. Vitoria-Gasteiz: Departamento de Medio Ambiente y Política Territorial del Gobierno Vasco.

Gobierno Vasco, 2018a. Aprobación inicial de la Revisión de las Directrices de Ordenación Territorial de la CAPV. BOPV 42, de 28 de febrero de 2018.

Gobierno Vasco, 2018b. Estrategia de Educación para la Sostenibilidad del País Vasco 2030. Vitoria-Gasteiz: Departamento de Medio Ambiente, Planificación Territorial y Vivienda del Gobierno Vasco.

Gobierno Vasco, 2018c. Azterkosta 2017. Programa Aztertu. Vitoria-Gasteiz: Departamento de Medio Ambiente, Planificación Territorial y Vivienda del Gobierno Vasco. 
Iglesias, B., Anfuso, G., Uterga, A., Arenas, P. y Williams, A.T., 2018. Scenic value of the Basque Country and Catalonia coasts (Spain): impacts of tourist occupation. Journal of Coastal Conservation, 22, 247-261. DOI: https://doi.org/10.1007/s11852-017-0570-0

Lazcano, I. y Arzoz, X., 2009. La ordenación, planificación y gestión del litoral en el País Vasco. En: Sanz, F.J. (dir) y García, M.M. (coord). Estudios sobre la ordenación, planificación y gestión del litoral: hacia un modelo integrado y sostenible. A Coruña: Fundación Pedro Barrié de la Maza, pp. 287-298.

Marcos, M., Chust, G., Jordà, G. y Caballero, A., 2012. Effect of sea level extremes on the western Basque coast during the 21st century. Climate Research, 51, 237-248. DOI: https://doi.org/10.3354/cr01069

Monge-Ganuzas, M., Cearreta, A. y García-Artola, A., 2019. Coastal management in the Basque coast: A case study of dredging and dumping operations along the Oka Estuary. En: Morales J. (ed.) The Spanish coastal systems. Cham: Springer, pp. 729-744.

DOI: https://doi.org/10.1007/978-3-319-93169-2

Parlamento Europeo y Consejo de la UE, 2002. Recomendación del Parlamento Europeo y del Consejo, de 30 de mayo de 2002, sobre la aplicación de la gestión integrada de las zonas costeras en Europa. Diario Oficial de las Comunidades Europeas, 148, de 6 de junio de 2002.

Parlamento Europeo y Consejo de la UE, 2014. Directiva 2014/89/UE del Parlamento Europeo y del Consejo de 23 de julio de 2014, por la que se establece un marco para la ordenación del espacio marítimo. Diario Oficial de la UE 257, de 28 de agosto de 2014.

Pérez-Cayeiro, M.L., 2013. Gestión Integrada de Áreas Litorales. Análisis de los fundamentos de la disciplina. Madrid: Tébar.

Porcal-Gonzalo, M.C., Díez, A. y Junguitu, J., 2012. Dimensión territorial y turística de la Ruta Norte del Camino de Santiago en el País Vasco: distintas concepciones, valoraciones y propuestas de intervención en un fenómeno multifacético. Boletín de la Asociación de Geógrafos Españoles, 58, 177-204.

Post, J.C. y Lundin, C.G. (eds), 1996. Guidelines for Integrated Coastal Zone Management. Washington: The World Bank. DOI: https://doi.org/10.1596/0-8213-3735-1

Puig, J. y Villarroya, A., 2013. Ecological quality loss and damage compensation in estuaries: Clues from a lawsuit in the Basque Country, Spain. Ocean \& Coastal Management, 71, 46-51. DOI: https://doi.org/10.1016/j.ocecoaman.2012.09.005

Sanò, M., González-Riancho, P., Areizaga, J. y Medina, R., 2010. The strategy for coastal sustainability: A Spanish initiative for ICZM. Coastal Management, 38, 76-96. DOI: https://doi.org/10.1080/08920750903411734

Torres-Delgado, A. y Saarinen, J., 2014. Using indicators to assess sustainable tourism development: a review. Tourism Geographies, 16, 31-47. DOI: https://doi.org/10.1080/14616688.2013.867530 
